\title{
Front Matter: Volume 6778
}

, "Front Matter: Volume 6778," Proc. SPIE 6778, Three-Dimensional TV, Video, and Display VI, 677801 (17 October 2007); doi: 10.1117/12.776208

SPIE. Event: Optics East, 2007, Boston, MA, United States 


\title{
PROCEEDINGS OF SPIE
}

\section{Three-Dimensional TV, Video, and Display VI}

\author{
Bahram Javidi \\ Fumio Okano \\ Jung-Young Son \\ Editors
}

11-12 September 2007

Boston, Massachusetts, USA

Sponsored and Published by

SPIE

Volume 6778 
The papers included in this volume were part of the technical conference cited on the cover and title page. Papers were selected and subject to review by the editors and conference program committee. Some conference presentations may not be available for publication. The papers published in these proceedings reflect the work and thoughts of the authors and are published herein as submitted. The publisher is not responsible for the validity of the information or for any outcomes resulting from reliance thereon.

Please use the following format to cite material from this book:

Author(s), "Title of Paper," in Three-Dimensional TV, Video, and Display VI, edited by Bahram Javidi, Fumio Okano, Jung-Young Son, Proceedings of SPIE Vol. 6778 (SPIE, Bellingham, WA, 2007) Article CID Number.

ISSN 0277-786X

ISBN 9780819469380

Published by

SPIE

P.O. Box 10, Bellingham, Washington 98227-0010 USA

Telephone +1 3606763290 (Pacific Time) · Fax +1 3606471445

SPIE.org

Copyright (c) 2007, Society of Photo-Optical Instrumentation Engineers

Copying of material in this book for internal or personal use, or for the internal or personal use of specific clients, beyond the fair use provisions granted by the U.S. Copyright Law is authorized by SPIE subject to payment of copying fees. The Transactional Reporting Service base fee for this volume is $\$ 18.00$ per article (or portion thereof), which should be paid directly to the Copyright Clearance Center (CCC), 222 Rosewood Drive, Danvers, MA 01923. Payment may also be made electronically through CCC Online at copyright.com. Other copying for republication, resale, advertising or promotion, or any form of systematic or multiple reproduction of any material in this book is prohibited except with permission in writing from the publisher. The CCC fee code is $0277-786 \mathrm{X} / 07 / \$ 18.00$.

Printed in the United States of America.

Publication of record for individual papers is online in the SPIE Digital Library.

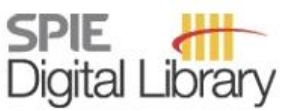

SPIEDigitalLibrary.org

Paper Numbering: Proceedings of SPIE follow an e-First publication model, with papers published first online and then in print and on CD-ROM. Papers are published as they are submitted and meet publication criteria. A unique, consistent, permanent citation identifier (CID) number is assigned to each article at the time of the first publication. Utilization of CIDs allows articles to be fully citable as soon they are published online, and connects the same identifier to all online, print, and electronic versions of the publication. SPIE uses a six-digit CID article numbering system in which:

- The first four digits correspond to the SPIE volume number.

- The last two digits indicate publication order within the volume using a Base 36 numbering system employing both numerals and letters. These two-number sets start with 00, 01, 02, 03, 04, 05, $06,07,08,09,0 \mathrm{~A}, 0 \mathrm{~B} \ldots \mathrm{OZ}$, followed by 10-1Z, 20-2Z, etc.

The CID number appears on each page of the manuscript. The complete citation is used on the first page, and an abbreviated version on subsequent pages. Numbers in the index correspond to the last two digits of the six-digit CID number. 


\section{Contents}

vii Conference Committee

\section{KEYNOTE PRESENTATIONS}

677803 Status and prospect of 3D/2D convertible displays (Keynote Paper) [6778-07]

B. Lee, H. Choi, Y. Kim, Seoul National Univ. (South Korea)

677804 Disparity-based quality function for autostereoscopic display devices (Keynote Paper) [6778-08]

J.-Y. Son, Daegu Univ. (South Korea); V. V. Saveljev, Hanyang Univ. (South Korea); M.-C. Park, Korea Institute of Science and Technology (South Korea); D.-S. Kim, Samsung Electronics Co., Ltd. (South Korea); S.-K. Kim, Korea Institute of Science and Technology (South Korea)

677805 Three-dimensional integral television using extremely high-resolution video system with 4,000 scanning lines (Keynote Paper) [6778-23]

F. Okano, M. Kawakita, J. Arai, H. Sasaki, T. Yamashita, NHK-Japan Broadcasting Corporation (Japan); M. Sato, K. Suehiro, Y. Haino, Victor Co. of Japan, Ltd. (Japan)

677806 Relay optics for enhanced integral imaging (Keynote Paper) [6778-24]

R. Martinez-Cuenca, G. Saavedra, Univ. of Valencia (Spain); B. Javidi, Univ. of Connecticut (USA); M. Martinez-Corral, Univ. of Valencia (Spain)

\section{SESSION 1 HOLOGRAPHY}

677807 Three-dimensional interface based on digital holography (Invited Paper) [6778-02]

O. Matoba, K. Nitta, Kobe Univ. (Japan)

677808 Real-time elimination of twin images in digital holographic microscope [6778-03] H. Cho, D. Kim, Y. Yu, Cheju National Univ. (South Korea); S. Shin, AP \& Tec (South Korea); W. Jang, Korea Photonics Technology Institute (South Korea); J.-Y. Son, Daegu Univ. (South Korea)

677809 Real-time automated three-dimensional recognition of micro/nano biological organisms [6778-04]

I. Moon, B. Javidi, Univ. of Connecticut (USA)

6778 0A Improved resolution synthetic aperture holographic imaging (Invited Paper) [6778-44]

L. Martínez-León, Univ. Jaume I (Spain); B. Javidi, Univ. of Connecticut (USA) 
6778 OB Monocular accommodation condition in 3D display types through geometrical optics (Invited Paper) [6778-06]

S.-K. Kim, D.-W. Kim, M.-C. Park, Korea Institute of Science and Technology (South Korea); J.-Y. Son, Daegu Univ. (South Korea)

6778 OC Ray-space acquisition system of all-around convergent views using a rotation mirror [6778-09]

K. Manoh, T. Yendo, T. Fujii, M. Tanimoto, Nagoya Univ. (Japan)

6778 OD Elimination of flipped image and enhancement of viewing angle for lenticular 3D display [6778-10]

S. Sakamoto, Y. Takaki, Tokyo Univ. of Agriculture and Technology (Japan)

6778 OE Resolution analysis of lenticular-sheet 3D display system [6778-11]

T. Saishu, K. Taira, Toshiba Corp. (Japan)

\section{SESSION 3 3D DISPLAY SYSTEM II}

6778 OF View synthesis for uncalibrated camera (Invited Paper) [6778-12]

J. Seo, J. Kang, K. Sohn, Yonsei Univ. (South Korea)

6778 OG Interactive 3D responsive virtual space service using mobile phone [6778-13]

Y.-M. Kwon, K.-W. Jeon, J. Ki, M. N. Mayangsari, Korea Institute of Science and Technology (South Korea)

$6778 \mathrm{OH}$ Constraints for stereoscopic vision in stereo camera phone [6778-14]

K.-H. Lee, Konkuk Univ. (South Korea) and Korea Institute of Science and Technology (South Korea); S.-H. Kim, Y.-S. Yoon, Konkuk Univ. (South Korea); J.-Y. Son, Daegu Univ. (South Korea); M.-C. Park, S.-K. Kim, Korea Institute of Science and Technology (South Korea)

6778 ol A networked viewpoint controller based on a spatiotemporal attention module for the future 3D TV [6778-15]

M.-C. Park, Korea Institute of Science and Technology (South Korea); W. Choi, McMaster Univ. (Canada); S. K. Kim, Korea Institute of Science and Technology (South Korea); J.-Y. Son, Daegu Univ. (South Korea)

6778 0J Single-shot compressive imaging [6778-16]

A. Stern, Y. Rivenson, Ben Gurion Univ. of the Negev (Israel); B. Javidi, Univ. of Connecticut (USA)

\section{SESSION 4 3D DISPLAY SYSTEM III}

6778 OK Time-multiplexing 3D display using fast light shutter [6778-17]

J. Yamamoto, T. Yendo, T. Fujii, M. Tanimoto, Nagoya Univ. (Japan) 
$67780 \mathrm{M}$ Distortion-tolerant object classification using photon counting three-dimensional integral imaging (Invited Paper) [6778-19]

S. Yeom, Daegu Univ. (South Korea); B. Javidi, Univ. of Connecticut (USA); E. Watson, Air Force Research Lab. (USA)

$6778 \mathrm{ON}$ Three-dimensional tracking of objects in holographic imaging [6778-20]

M. DaneshPanah, B. Javidi, Univ. of Connecticut (USA)

$677800 \quad$ Analysis of synthetic aperture integral imaging [6778-21]

B. Tavakoli, M. DaneshPanah, B. Javidi, Univ. of Connecticut (USA); E. Watson, Air Force Research Lab. (USA)

6778 OP Multifocus holographic 3D image fusion using independent component analysis [6778-22] C. M. Do, B. Javidi, Univ. of Connecticut (USA)

\section{SESSION 5 INTEGRAL IMAGING I}

$67780 Q \quad$ Implementation of a novel floating-image display system having a background of multiview integral images (Invited Paper) [6778-25]

S.-P. Hong, Y.-S. Oh, Kwangwoon Univ. (South Korea); D.-H. Shin, Dongseo Univ. (South Korea); E.-S. Kim, Kwangwoon Univ. (South Korea)

6778 OR Efficient reduction of defocused areas in the reconstructed image by computational integral imaging reconstruction [6778-26]

K.-J. Lee, C.-K. Kim, D.-C. Hwang, E.-S. Kim, Kwangwoon Univ. (South Korea)

6778 OS Three-dimensional imaging using randomly-distributed cameras on the circle [6778-27]

S. Maehara, O. Matoba, K. Nitta, T. Yoshimura, Kobe Univ. (Japan)

\section{SESSION 6 INTEGRAL IMAGING II}

6778 OT New 3D display using lens array and depth division images [6778-28]

A. Takeichi, T. Yendo, T. Fujii, M. Tanimoto, Nagoya Univ. (Japan)

6778 OU Improved viewing resolution of three-dimensional integral imaging using pinhole array on LC panel [6778-29]

Y. Kim, J. Kim, J.-M. Kang, J.-H. Jung, B. Lee, Seoul National Univ. (South Korea)

\section{SESSION 7 IMAGE PROCESSING}

6778 OY 3D video system for capturing unexpected moments in daily life [6778-33]

H. Kim, R. Sakamoto, Advanced Telecommunications Research Institute (Japan); I. Kitahara, Advanced Telecommunications Research Institute (Japan) and Univ. of Tsukuba (Japan); T. Toriyama, K. Kogure, Advanced Telecommunications Research Institute (Japan)

$67780 Z$ 3D TV by holographic stereogram (II) [6778-05]

K. Sato, L. Tian, H. Zhao, Shonan Institute of Technology (Japan); K. Takano, Tokyo Metropolitan College of Industrial Technology (Japan) 
677813 A global disparity adjustment scheme using binocular energy model and image partition [6778-37]

Y. Park, Korea Univ. of Science and Technology (South Korea); N. Hur, Electronics and Telecommunications Research Institute (South Korea)

677815 Holographic 3D display of picked up image by integral imaging technique [6778-39] S.-H. Lee, S.-C. Kim, E.-S. Kim, Kwangwoon Univ. (South Korea)

677816 Transmission of holographic 3D images using infrared transmitter (II): on a study of transmission of holographic 3D images using infrared transmitter safe to medical equipment [6778-40]

K. Takano, K. Muto, Tokyo Metropolitan College of Industrial Technology (Japan); L. Tian, K. Sato, Shonan Institute of Technology (Japan)

677817 Depth-fused 3D (DFD) display with multiple viewing zones [6778-42]

M. Date, S. Sugimoto, H. Takada, K. Nakazawa, Nippon Telegraph and Telephone Corp. (Japan)

677818 Image stitch-based multiview video coding [6778-43]

K. Sohn, Yonsei Univ. (South Korea); Y. Kim, Samsung Electronics Co., Ltd. (South Korea);

H. Ko, J. Seo, Yonsei Univ. (South Korea)

Author Index 


\title{
Conference Committee
}

\author{
Symposium Chairs \\ Achyut Kumar Dutta, Banpil Photonics, Inc. (USA) \\ Werner Weiershausen, T-Systems Enterprise Services GmbH (Germany) \\ Conference Chairs \\ Bahram Javidi, University of Connecticut (USA) \\ Fumio Okano, NHK Science and Technical Research Laboratories \\ (Japan) \\ Jung-Young Son, Daegu University (South Korea) \\ Program Committee \\ Rene De la Barre, Fraunhofer Institute for Telecommunication \\ (Germany) \\ Toshiaki Fujii, Nagoya University (Japan) \\ William E. Higgins, Pennsylvania State University (USA) \\ Manuel Martínez-Corral, Universitat de València (Spain) \\ Osamu Matoba, Kobe University (Japan) \\ Thomas J. Naughton, National University of Ireland, Maynooth (Ireland) \\ Takanori Nomura, Wakayama University (Japan) \\ Wa James Tam, Communications Research Center Canada \\ (Canada)

\section{Session Chairs} \\ 1 Holography \\ George Barbastathis, Massachusetts Institute of Technology (USA) \\ 2 3D Display System I \\ Byoungho Lee, Seoul National University (South Korea) \\ 3 3D Display System II \\ Min-Chul Park, Korea Institute of Science and Technology (South \\ Korea) \\ 4 3D Display System III \\ Tomohiro Yendo, Nagoya University (Japan) \\ $5 \quad$ Integral Imaging I \\ Fumio Okano, NHK Science and Technical Research Laboratories \\ (Japan)
}


6 Integral Imaging II

Jung-Young Son, Daegu University (South Korea)

$7 \quad$ Image Processing

Osamu Matoba, Kobe University (Japan) 